\title{
Obsessive-Compulsive Disorder (OCD) with and Without Psychotic Symptoms; Psychopathological and Neuroimaging Aspects: Case-Control Study
}

Amany Ibrahim Falah ${ }^{1}$,Tarek Ahmed Okasha ${ }^{2}$, Nermin Mahmoud Shaker ${ }^{2}$, Reem Elsayed Mohamed Hashem ${ }^{2}$, Yousra Abdel Zaher Abdallah ${ }^{3}$

Ministry of health, Egypt ${ }^{1}$, Institute of Psychiatry, Ain Shams University ${ }^{2}$, Radiology department, Faculty of Medicine, Ain Shams University, Egypt ${ }^{3}$

\section{BACKGROUND}

- OCD can have disabling effects throughout the patient's lifespan. (Attiullah et al., 2000).

- It is crucial to differentiate between OCD and a psychotic disorder, because some antipsychotics may induce or exacerbate symptoms of OCD (Singer, 2014).

- Despite this phenotypic heterogeneity, standard nomenclatures (DSM-IV and ICD-10) regard OCD as a unitary nosological entity, while this parsimony has some esthetic appeal, it may be misleading.

- Neuroimaging studies have the potential to increase our understanding of the connection between observable symptoms and associated neurobiology, and perhaps lead to improvements in treatment and in matching treatment to patient needs (Rauch and Saxena, 2000).

\section{METHODS}

- The clinical sample of this study consisted of a convenient sample of 100 patients diagnosed with OCD. - Patients were divided into two groups: OCD patients with psychotic symptoms (B) and without psychotic symptoms(A).

- Patients were subjected to SCID I, SCID II, YBOCS, PANSS, BSRS.

-60 DTI Scans were included in the neuroimaging analysis by using TBSS, comparing 30 patients from each group and both groups were compared to a matched control group.

\section{FURTHER INFORMATION}

Dr. Amany Ibrahim Falah Consultant Psychiatrist, Ministry of health, Cairo, Egypt.

Amanyfalah@hotmail.com

\section{RESULTS}

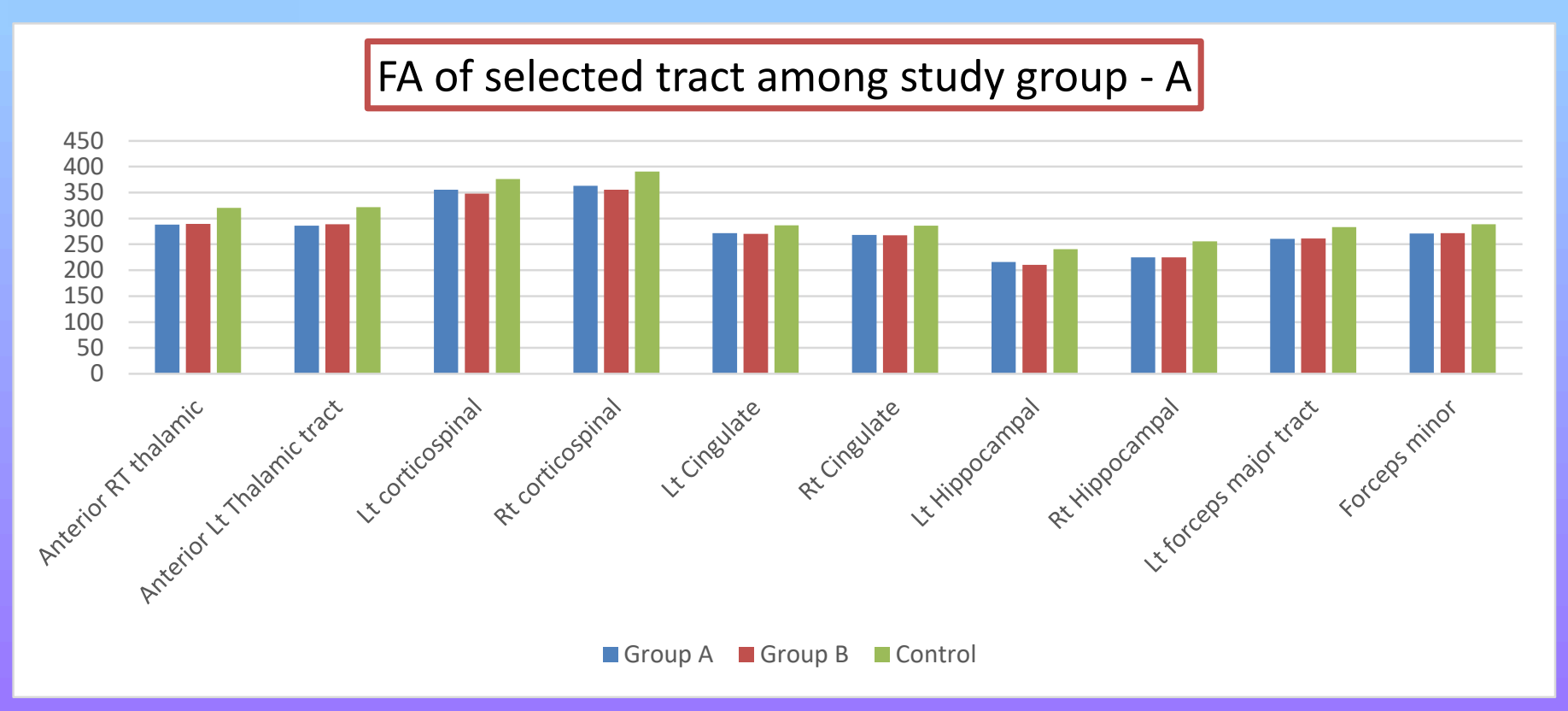

Fig (1): FA results of the selected tract among study group. Group A (OCD only) \& Group B (OCD with psychotic features)

\section{FA results among study group - $B$}

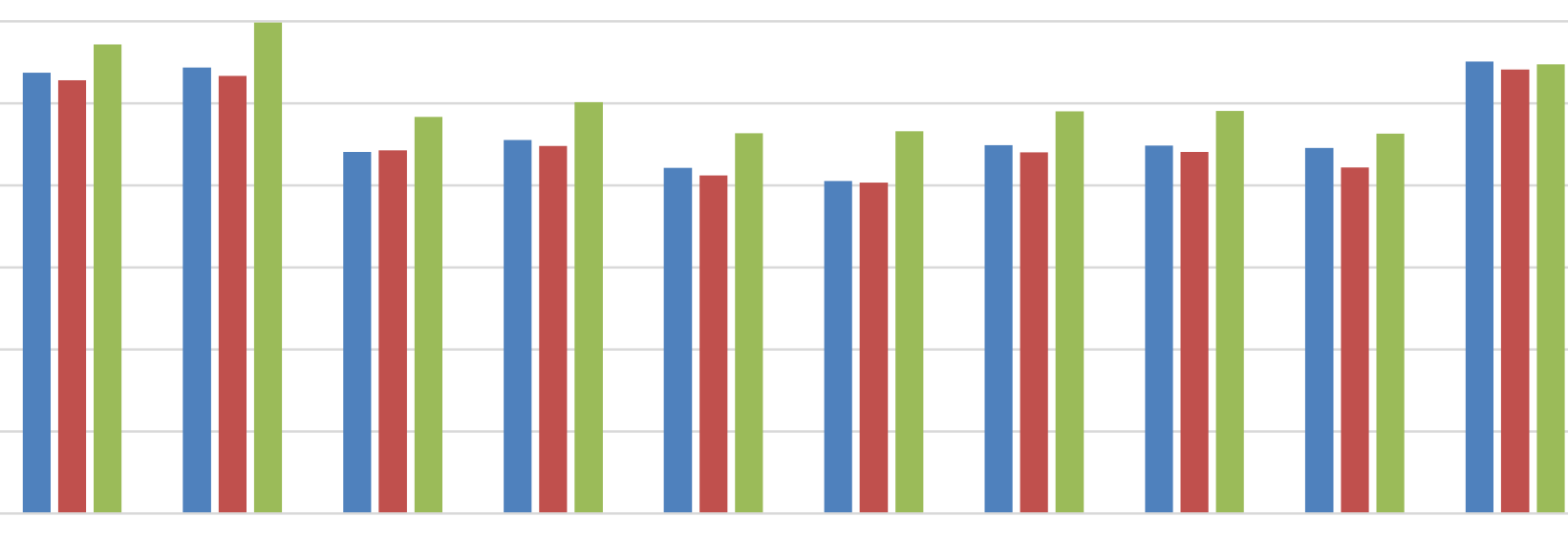

Fig (2): FA results of the selected tract among study group- continued.

\begin{tabular}{|c|c|c|c|c|c|c|c|c|c|}
\hline \multirow[t]{2}{*}{ volume of tracts } & \multicolumn{2}{|c|}{ OCD } & \multicolumn{2}{|c|}{$\begin{array}{c}\text { OCD with } \\
\text { psychotic features }\end{array}$} & \multicolumn{2}{|c|}{ Control } & \multirow[t]{2}{*}{ P1 } & \multirow[t]{2}{*}{ P2 } & \multirow[t]{2}{*}{ P3 } \\
\hline & Mean & SD & Mean & SD & Mean & SD & & & \\
\hline Anterior RT thalamic & 60911 & 13045.3 & 54424.7 & 6592.3 & 52835.5 & 4858.2 & $0.01 *$ & 0.05 * & 0.4 \\
\hline Anterior Lt Thalamic & 53658.1 & 9237.8 & 49496.5 & 5825.8 & 42427.2 & 3823.2 & $0.04 *$ & 0.000 ** & 0.001 ** \\
\hline Lt corticospinal & 46488.7 & 6256.4 & 42472.1 & 5122.1 & 37060.5 & 3172.9 & $0.009 * *$ & 0.000 ** & $0.002 * *$ \\
\hline Rt corticospinal & 45941.4 & 7408.6 & 41668.6 & 4091.9 & 35867.7 & 2250.9 & $0.008 * *$ & $0.000 * *$ & $0.000 * *$ \\
\hline Lt Cingulate & 27969.1 & 3447.8 & 25566.1 & 2688.4 & 22557.4 & 3167.5 & 0.004 ** & 0.000 ** & $0.004 * *$ \\
\hline Rt Cingulate & 21833.7 & 2537.7 & 20110.6 & 2522.3 & 15530.8 & 2285.6 & $0.01 *$ & 0.000 *** & $0.000 * *$ \\
\hline Lt Hippocampal & 9220.1 & 1104.7 & 8532 & 1446.3 & 8021.8 & 959 & $0.04 *$ & $0.003 * *$ & 0.2 \\
\hline Rt Hippocampal & 9564.1 & 1188 & 8640.9 & 1141.9 & 8140.4 & 1146 & $0.003 * *$ & 0.001 ** & 0.2 \\
\hline Lt forceps major & 56634.5 & 8333.2 & 48625.2 & 7416 & 46372.3 & 6156.1 & $0.000 * *$ & 0.001 ** & 0.3 \\
\hline Forceps minor & 46477.4 & 5238.9 & 43798.6 & 4182.7 & 40200.9 & 3808.5 & $0.03 *$ & 0.001 ** & $0.01 *$ \\
\hline Lt Inferior fronto occipital & 41575.4 & 4471 & 38711 & 3642.4 & 38405.4 & 3322.5 & 0.009 ** & $0.03 *$ & 0.8 \\
\hline Rt Inferior fronto occipital & 57697.5 & 6098.7 & 53583.4 & 5619.2 & 48518.6 & 5397.6 & 0.009 ** & 0.000 *** & $0.01^{*}$ \\
\hline Lt Inferior long fasciulus & 59804.6 & 6416.8 & 57534.1 & 5609.8 & 44541.4 & 4645.4 & 0.1 & 0.000 ** & $0.000^{* * *}$ \\
\hline Rt inferior long fasciulus & 51395.8 & 6798.2 & 48776.3 & 5215.5 & 39161.1 & 3874.1 & 0.09 & 0.000 ** & $0.000^{* * *}$ \\
\hline Lt superior long fasiculus & 127773 & 15855.4 & 118221.3 & 13127.8 & 90406.1 & 9242.1 & 0.01 * & 0.000 ** & $0.000^{* * *}$ \\
\hline Rt superior long fasiculus & 126135.8 & 13248.8 & 114788.9 & 12124.1 & 92065.5 & 7450.5 & 0.001 ** & $0.000^{* *}$ & $0.000^{* * *}$ \\
\hline Lt Uncinate & 8655.5 & 1145.1 & 8769.2 & 1087.7 & 9453.7 & 853.3 & 0.6 & $0.04 *$ & $0.06^{*}$ \\
\hline Rt uncinate & 8159 & 910.7 & 7873.3 & 853 & 6979.7 & 687.8 & 0.2 & $0.000^{* * *}$ & $0.003 * *$ \\
\hline Lt longitudinal fasiculus & 1820.8 & 259.1 & 1724.2 & 291.5 & 1229.5 & 186.1 & 0.1 & $0.000^{* *}$ & $0.000^{* * *}$ \\
\hline Rt long fasiculus & 5531.1 & 843.4 & 4951.5 & 877.3 & 4898.4 & 572.6 & $0.01 *$ & $0.02 *$ & 0.8 \\
\hline
\end{tabular}

Table (1): Volume results in selected tracts among the study group.

P1 correlation between Group A (OCD only) \& Group B (OCD with psychotic features); P2 correlation between Group (OCD only) \& control; p3 correlation between group B (OCD with psychotic features) \& control
RESULTS CONTINUED

- Comparison between the two groups using DTI (FA of the ROI and selected tracts) showed that: -right hippocampal showed statistical significant difference across all groups

-left uncinate tract showed affection among OCD patient with psychotic features in comparison to control only while it didn't affect OCD patients without psychotic features.

- There was statistical significant difference between the volume of tracts ( anterior right \& left thalamic, right \& left corticospinal, right \& left cingulate, right \& left hippocampal, forceps major \& minor, right \& left inferior fronto occipital, right \& left superior fasiculus, \& right long fasiculus) between the OCD only group \& OCD with psychotic features.

\section{CONCLUSIONS}

- Patients suffering from OCD with psychotic symptoms have neuroimaging aspects different from other OCD patients which makes them distinct subgroup.

- Taking into consideration, the need to add a specific specifier in the diagnostic classification which means that those groups of patients need a specific plan of management.

\section{APPENDIX}

- BSRS: brief psychiatric rating scale

- DTI: diffuse tensor imaging

- FA: fractional anisotropy

- PNSS: positive and negative syndrome scale

- ROI: region of interest

- SCID: structured clinical interview for DSM

-Y.bocs: Yale brown obsessive compulsive scale

\section{REFERENCES}

1. Attiullah N, Eisen JL, Rasmussen SA (2000): Clinical features of obsessive compulsive disorder. Psychiatr cli North Am 2000, 23:469-491. 2. Singer, J. (2014): The Connection between OCD \& Psychosis. Psych Central. Retrieved on July 6, 2014, from 